\title{
ATIVIDADE INSETICIDA DOS FRUTOS DE Trichilia claussenii (MELIACEAE) SOBRE Spodoptera frugiperda
}

\author{
Liliane Nebo, Andréia Pereira Matos, Paulo Cezar Vieira*, João Batista Fernandes e Maria Fátima das Graças \\ Fernandes da Silva \\ Departamento de Química, Universidade Federal de São Carlos, CP 676, 13565-905 São Carlos - SP, Brasil \\ Ricardo Ribeiro Rodrigues \\ Departamento de Ciências Biológicas, Escola Superior de Agricultura “Luiz de Queiroz”, Universidade de São Paulo, CP 9, \\ 13418-900 Piracicaba - SP, Brasil
}

Recebido em 29/11/09; aceito em 1/6/10; publicado na web em 24/8/10

\begin{abstract}
INSECTICIDAL ACTIVITY OF Trichilia claussenii (MELIACEAE) FRUITS AGAINST Spodoptera frugiperda. An evaluation of the insecticidal activity of the fruits extracts of Trichilia claussenii was carried out and the methanol extract revealed to have strong insecticidal activity. The fractionation of methanol extract of T. claussenii seeds bioassay-guided against Spodoptera frugiperda has led to the identification of the $\omega$-phenylalkyl and alkenyl fatty acids as active compounds in this extract. The structures of the compounds were proposed by spectroscopic analysis and comparison with literature data.
\end{abstract}

Keywords: Trichilia claussenii; $\omega$-phenylalkyl and alkenyl fatty acids; Spodoptera frugiperda.

\section{INTRODUÇÃO}

O milho representa um dos principais cereais em todo mundo e é cultivado em pequenas, médias e grandes propriedades. No Brasil é considerada como cultura de expressão nacional, de grande importância social e econômica, e está presente de norte a sul do País. ${ }^{1}$ São utilizados, durante o desenvolvimento da cultura, insumos sintéticos como pesticidas que, além de aumentar os custos de produção, poluem o ambiente e deixam resíduos nos alimentos. O controle de pragas é uma das atividades de importância econômica que deve ser bem planejada para a obtenção de maiores rendimentos econômicos. ${ }^{2}$ A lagarta-do-cartucho, Spodoptera frugiperda (J. E. Smith), destaca-se como importante praga do milho, tanto pela redução da produtividade e da qualidade do produto final, quanto pela dificuldade de controle..$^{2,3}$

Um dos primeiros relatos da atividade inseticida de Trichilia spp. sobre S. frugiperda foi feito por Mikolajczak e Reed. ${ }^{4}$ Experimentos realizados adicionando extratos aquosos de T. casaretti, T. catigua, T. claussenii, T. elegans e T. pallida à dieta artificial afetaram o desenvolvimento das lagartas de $S$. frugiperda. ${ }^{5}$ Extratos orgânicos das folhas, ramos e frutos de T. claussenii e dos frutos de T. catigua, T. elegans foram avaliados sobre $S$. frugiperda em condições de laboratório. ${ }^{6,7}$ Os extratos hexânico e metanólico de folhas e o hexânico de ramos de T. claussenii foram os mais eficientes, apresentando alta taxa de mortalidade larval (superior a $60,0 \%) .{ }^{6}$ Dentre os frutos, os melhores resultados foram apresentados pelos extratos hexânico e metanólico de T. elegans com mortalidade larval de $100,0 \%$. $^{7}$

O estudo químico das folhas de T. claussenii resultou no isolamento de um cicloartano, uma mistura de ácidos $\omega$-fenil alcanoicos e alquenoicos, aminoácidos e um sesquiterpeno ${ }^{8}$ dos galhos foram isolados esteroides pregnanos e sesquiterpenos ${ }^{9} \mathrm{e}$, dos frutos, novas $\gamma$-lactonas. ${ }^{10}$

Em vista do exposto, desenvolveu-se este trabalho com o objetivo de avaliar a bioatividade de extratos orgânicos e dos constituintes químicos dos frutos de $T$. claussenii sobre $S$. frugiperda.

*e-mail: paulo@dq.ufscar.br

\section{PARTE EXPERIMENTAL}

\section{Procedimentos experimentais gerais}

Os espectros de RMN de ${ }^{1} \mathrm{He} \mathrm{e}^{13} \mathrm{C}$ foram obtidos em espectrômetro Bruker DRX operando a $400 \mathrm{MHz}$ para ${ }^{1} \mathrm{H}$, utilizando-se $\mathrm{CDCl}_{3}$ como solvente e TMS como padrão interno.

As separações cromatográficas em colunas foram realizadas utilizando-se gel de sílica 60, 70-230, 230-400 mesh. As análises cromatográficas em camada fina foram realizadas em cromatoplacas de sílica gel $\mathrm{F}_{254}$ sobre placa de alumínio Merck, de 0,2 mm de espessura, empregando-se como revelador a solução de vanilina/ácido sulfúrico.

Para identificação dos ésteres metílicos do conjunto dos ácidos $\omega$-fenil alcanoicos e alquenoicos foi utilizado cromatógrafo gasoso acoplado ao espectrômetro de massas, marca Shimadzu, modelo QP-5000, com coluna capilar DB-5 (30 m x 0,25 mm x 0,15 $\mu \mathrm{m})$, utilizando as seguintes condições: temperatura do detector: $280{ }^{\circ} \mathrm{C}$, injetor: $250{ }^{\circ} \mathrm{C}$, gás de arraste: He, temperatura inicial do forno: $70{ }^{\circ} \mathrm{C}$ por $2 \mathrm{~min}$, velocidade de aquecimento a $10^{\circ} \mathrm{C} / \mathrm{min}$ até $290^{\circ} \mathrm{C}$, permanecendo nessa temperatura por $20 \mathrm{~min}$. $\mathrm{O}$ espectro de massas foi obtido por impacto de elétrons a $70 \mathrm{eV}$.

\section{Material vegetal}

Os frutos de T. claussenii foram coletados em Piracicaba/SP no Campus da Escola Superior de Agricultura "Luiz de Queiroz"/USP, e identificados pelo botânico Dr. R. R. Rodrigues. Uma exsicata foi depositada no herbário da Escola Superior de Agricultura "Luiz de Queiroz" sob o número 7395.

\section{Preparação dos extratos}

Os frutos de T. claussenii foram secos em estufa de circulação de ar a $40{ }^{\circ} \mathrm{C}$, por aproximadamente 7 dias e posteriormente separados em cascas do fruto (pericarpo, TCCF, 56,8 g), cascas da semente (arilo, TCCS, 7,90 g), semente (TCS, 35,1 g). A extração das partes dos frutos de $T$. claussenii foi realizada por maceração a temperatura ambiente e em repouso, com solventes em ordem crescente de

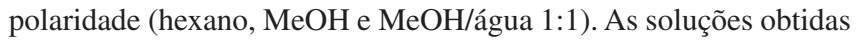


foram concentradas por destilação do solvente em evaporador rotativo, fornecendo os extratos hexânico, metanólico e hidrometanólico, respectivamente. Os extratos foram submetidos a ensaios de toxicidade sobre a lagarta-do-cartucho do milho, S. frugiperda, e os que apresentaram atividade foram fracionados.

\section{Ensaio de toxicidade}

Os ensaios biológicos sobre $S$. frugiperda foram realizados no Laboratório de Bioensaios do Departamento de Química da UFSCar, a $25 \pm 1{ }^{\circ} \mathrm{C}$, UR de $70 \pm 5 \%$ e fotofase de $12 \mathrm{~h}$. Para realização dos testes, a criação de $S$. frugiperda (J. E. Smith) foi mantida em laboratório, sendo as lagartas alimentadas com dieta artificial e os adultos com solução de mel a $10 \% .^{11}$

Os ensaios de toxicidade basearam-se na metodologia descrita por Paula et al.. ${ }^{12}$ Para realização dos bioensaios, foram preparadas soluções das amostras em acetona e/ou água. Foram utilizados grupos de 10 larvas de $S$. frugiperda no segundo instar (5 dias), onde cada grupo foi transferido para placas de Petri. As médias dos pesos dos grupos de insetos foram obtidas pelas medidas realizadas em balança analítica. Em cada inseto, $1 \mu \mathrm{L}$ da solução em acetona ou água foi aplicado topicamente, via uma microsseringa. Para evitar a possível morte do inseto, em cada grupo de larvas foi colocada pequena porção $(300,0$ $\mathrm{mg}$ ) da dieta artificial. Este processo foi realizado $1 \mathrm{~h}$ após a aplicação da solução. O controle consistiu na aplicação de $1 \mu \mathrm{L}$ de acetona ou água em cada inseto e foi realizado utilizando as mesmas condições descritas anteriormente. Todos os experimentos e o respectivo controle foram realizados em triplicata. Todos os ensaios foram realizados na concentração de $10 \mathrm{mg} / \mathrm{mL}$ e a mortalidade dos insetos foi verificada após $48 \mathrm{~h}$. Os resultados foram expressos em média da porcentagem da mortalidade e analisados utilizando-se a análise de variância (ANOVA), sendo usado $\mathrm{P} \leq 0,05$ como nível de significância.

\section{Isolamento e identificação dos constituintes químicos de $T$. claussenii}

O extrato metanólico das sementes de T. claussenii (TCSM, $4,54 \mathrm{~g}$ ) foi suspenso em uma mistura $\mathrm{MeOH} / \mathrm{H}_{2} \mathrm{O}$ (7:3) e particionado sucessivamente com hexano, $\mathrm{CH}_{2} \mathrm{Cl}_{2}$, AcOEt e $\mathrm{MeOH} / \mathrm{H}_{2} \mathrm{O}$, obtendo-se após a evaporação dos solventes as frações: TCSM1 $=0,37 \mathrm{~g} ;$ TCSM2 $=0,06 \mathrm{~g} ;$ TCSM3 $=1,00 \mathrm{~g}$ e TCSM4 $=3,10 \mathrm{~g}$, respectivamente. As frações obtidas foram submetidas ao ensaio de toxicidade sobre $S$. frugiperda. A fração bioativa TCSM1 $(0,37 \mathrm{~g})$ foi fracionada em coluna de gel de sílica (70-230 mesh) empregando-se como eluentes: hexano 100\%, hexano/AcOEt (99:1/98:2/95:5/9:1/8: 2/7:3/1:1), AcOEt 100\%, AcOEt/MeOH (99:1/98:2/95:5/9:1/8:2/7:3 /1:1) e MeOH 100\%; as frações obtidas foram reunidas em 6 frações (TCSM1-1 a TCSM1-6) com base na semelhança revelada pela análise por cromatografia em camada fina e, posteriormente, submetidas ao ensaio de toxicidade. As sub-frações bioativas TCSM1-3 (0,05 g) e TCSM1-4 $(0,20 \mathrm{~g})$ foram fracionadas em gel de sílica (70-230 mesh), utilizando como eluente hexano/AcOEt/MeOH (hexano/ AcOEt (8:2/7:3/1:1), AcOEt 100\%, AcOEt/MeOH (99:1/98:2/95:5/9: 1/8:2/7:3/1:1) e MeOH 100\%) em gradiente de polaridade crescente. Através das similaridades cromatográficas as frações foram reunidas e submetidas ao ensaio de toxicidade. As sub-frações biotivas TCSM1-3-2 (0,006 g) e TCSM1-4-4 (0,01 g) foram identificadas como mistura dos ácidos $\omega$-fenil alcanoicos e alquenoicos através da análise dos espectros de RMN de ${ }^{1} \mathrm{H}$ e por comparação com os dados da literatura. Para identificação do tamanho das cadeias metilênicas dos ácidos, uma pequena alíquota das amostras foi esterificada com diazometano como descrito por Leonard et al.,${ }^{13}$ fornecendo os ésteres metílicos identificados via CG/EM e por comparação com a literatura.

\section{RESULTADOS E DISCUSSÃO}

Inicialmente, os extratos orgânicos das cascas dos frutos (TCCF, pericarpo), cascas das sementes (TCCS, arilo) e sementes (TCS) de $T$. claussenii foram submetidos a ensaios de toxicidade sobre $S$. frugiperda. Os resultados iniciais mostraram que o extrato metanólico das sementes de T. claussenii (TCSM) provocou a mais alta porcentagem de mortalidade $(64,0 \%$, Figura 1$)$.

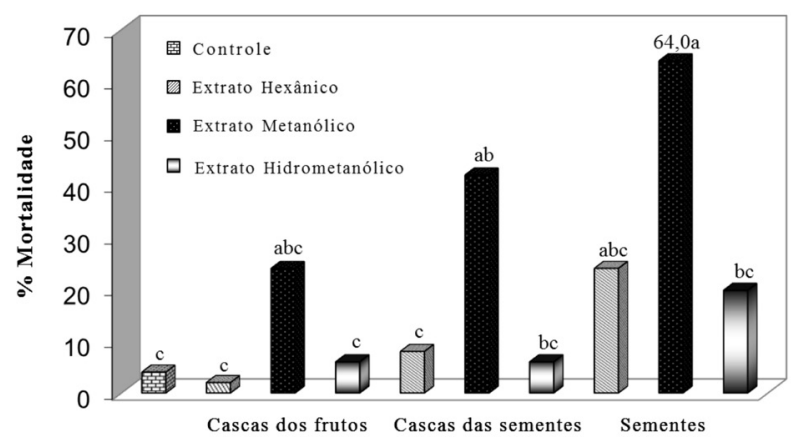

Figura 1. Ensaio de toxicidade realizado com os extratos dos frutos de T. claussenii sobre S. frugiperda na concentração de $10,0 \mathrm{mg} / \mathrm{mL}$

O extrato TCSM foi então fracionado, fornecendo as frações hexano (TCSM-1), diclorometano (TCSM-2), acetato de etila (TCSM-3) e hidrometanólico (TCSM-4). Entretanto, somente a fração TCSM-1 apresentou atividade relevante com 70,0\% de mortalidade sobre a lagarta-do-cartucho (Tabela 1).

Tabela 1. Resultados dos ensaios de toxicidade sobre $S$. frugiperda do extrato, frações e do conjunto dos ácidos $\omega$-fenil alcanoicos e alquenoicos de T. claussenii

\begin{tabular}{lc}
\hline Extrato & \% Mortalidade \\
TCSM & $64,0 \mathrm{a}$ \\
Frações & \\
TCSM-1 & $70,0 \mathrm{a}$ \\
TCSM-2 & $24,8 \mathrm{bc}$ \\
TCSM-3 & $26,7 \mathrm{bc}$ \\
TCSM-4 & $43,3 \mathrm{ab}$ \\
Substâncias & \\
Conjunto dos Ácidos $\omega$-fenil alcanoicos e & $73,3 \mathrm{a}$ \\
alquenoicos da fração TCSM-1-4-4 & \\
Controle & $6,60 \mathrm{c}$ \\
\hline
\end{tabular}

*Médias seguidas da mesma letra não diferem entre si pelo Teste de Tukey $(\mathrm{P} \leq 0,05)$

O fracionamento biomonitorado da fração TCSM-1 levou às subfrações bioativas TCSM-1-3-2 (59,0\% de mortalidade) e TCSM-1-4-4 (73,3\% de mortalidade) (Tabela 1$)$.

Os espectros de RMN de ${ }^{1} \mathrm{H}$ das frações TCSM-1-4-4 e TCSM1-3-2 evidenciaram a presença de ácidos $\omega$-fenil alcanoicos e alquenoicos nestas frações através dos sinais observados entre $\delta_{\mathrm{H}} 7,15-7,28$ (m) indicativos de hidrogênios ligados aos anéis aromáticos; dois tripletos em $\delta_{\mathrm{H}} 2,59$ e $\delta_{\mathrm{H}} 2,24$ referente aos hidrogênios das posições benzílicas e $\alpha$-carbonílicos, respectivamente; sinais em $\delta_{\mathrm{H}} 1,20-2,00$ (m) característicos de hidrogênios de cadeia metilênica e um sinal em $\delta_{H} 5,34(t)$ referente aos hidrogênios ligados às ligações duplas. A ausência de sinais entre $\delta_{\mathrm{H}} 3,50$ e $\delta_{\mathrm{H}} 4,50$ indica que não estão 
presentes nestas frações ésteres metílicos e/ou ésteres graxo. Esses dados para os ácidos $\omega$-fenil alcanoicos e alquenoicos são equivalentes aos relatados anteriormente. ${ }^{8,14}$

Para identificação dos constituintes minoritários (ácidos graxos), do tamanho das cadeias metilênicas dos ácidos $\omega$-fenil alcanoicos e alquenoicos e localização da posição da ligação dupla dos últimos, uma pequena alíquota das frações TCSM-1-4-4 e TCSM-1-3-2 foi esterificada com diazometano ${ }^{13} \mathrm{e}$, posteriormente, analisada via CG-EM. Através da comparação dos espectros de massas com os dados da biblioteca do CG-EM e da literatura, ${ }^{8,14}$ foi possível propor os tamanhos das cadeias metilênicas desses ácidos que variaram entre 10 a 15 carbonos (Figura 2, Tabela 2). Foi possível propor também a localização da ligação dupla dos ácidos $\omega$-fenil alquenoicos entre os carbonos $\delta$ - $\varepsilon$ em relação ao grupo fenila. Os ácidos $\omega$-fenil alquenoicos apresentaram como pico base $m / z, 104$, oriundo da fragmentação do tipo McLafferty da ligação dupla (Esquema 1). O cromatograma da fração bioativa TCSM-1-4-4 apresentou 39 picos, cujos espectros de massas foram comparados aos da biblioteca de dados do CG-EM e literatura, ${ }^{8,14}$ resultando na identificação de 18 ácidos que correspondem a 88,9\% da porcentagem relativa dos mesmos na fração, sendo $74,8 \%$ ácidos $\omega$-fenil alcanoicos e alquenoicos e $14,1 \%$ ácidos graxos (Tabela 2).

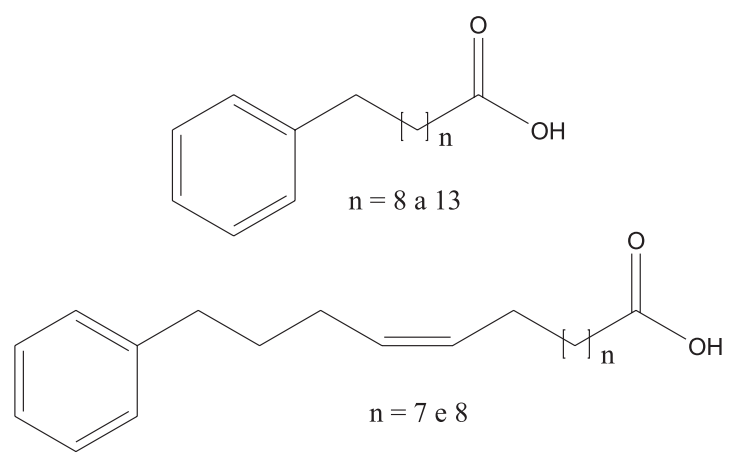

Figura 2. Ácidos $\omega$-fenil alcanoicos e alquenoicos identificados nas sementes de T. claussenii

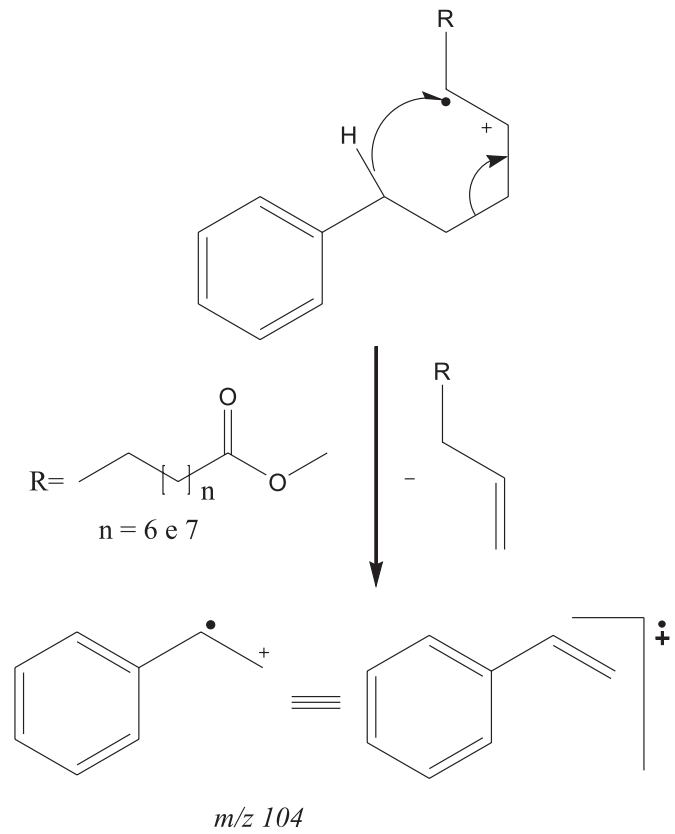

Esquema 1. Proposta de fragmentação do tipo McLafferty para ligações duplas em ácidos $\omega$-fenil alquenoicos
Tabela 2. Percentagem dos ésteres metílicos dos ácidos $\omega$-fenil alcanoicos, alquenoicos e graxos presentes na fração bioativa TCSM-1-4-4

\begin{tabular}{lcc}
\hline Ésteres & $\begin{array}{c}\text { Tempo de retenção } \\
(\mathrm{min})\end{array}$ & $\begin{array}{c}\text { Percentagem } \\
(\%)\end{array}$ \\
\hline Octanodioato de metila & 12,361 & 0,66 \\
Nonanodioato de metila & 13,713 & 0,86 \\
Tetradecanoato de metila & 15,948 & 0,60 \\
Pentadecanoato de metila & 17,107 & 1,48 \\
Hexadecenoato de metila & 17,949 & 0,27 \\
Hexadecanoato de metila & 18,217 & 5,95 \\
Heptadecenoato de metila & 19,017 & 2,50 \\
$\omega$-fenil decanoato de metila & 19,242 & 1,24 \\
Octadecenoato de metila & 20,029 & 1,17 \\
$\omega$-fenil undecanoato de metila & 20,295 & 4,20 \\
& & 14,16 \\
$\omega$-fenil dodecanoato de metila & 21,348 & 18,51 \\
$\omega$-fenil tridecanoato de metila & 22,317 & 18,51 \\
$\omega$-fenil tetradecenoato de metila & 22,953 & 3,37 \\
$\omega$-fenil tetradecanoato de metila & 23,222 & 12,84 \\
$\omega$-fenil pentadecenoato de metila & 23,848 & 5,18 \\
Docosanoato de metila & 23,913 & 0,27 \\
$\omega$-fenil pentadecanoato de metila & 24,131 & 15,30 \\
Tetracosanoato de metila & 25,755 & 0,34 \\
Total & - & 88,9 \\
\hline
\end{tabular}

A ocorrência dos ácidos $\omega$-fenil alcanoicos e alquenoicos já foi relatada anteriormente em folhas de $T$. claussenii, ${ }^{8}$ porém ainda não havia relatos da associação dos mesmos com a atividade inseticida encontrada nesta espécie.

Vanderherchen et al. ${ }^{15}$ sintetizaram substâncias da classe de ácidos $\omega$-fenil alcanoicos e demonstraram a atividade dos mesmos em outras espécies de insetos. Ensaios por aplicação tópica foram realizados com os ácidos 7-fenileptanoico e 7-fenilept-4-enoico na dose de 50,0 $\mu \mathrm{g} / \mathrm{inseto}$ em lagartas de $4^{\circ}$ instar de Manduca sexta (Lepdoptera: Sphingidae), Heliothis virescens (Lepdoptera: Noctuidae) e Helicoverpa zea (Lepdoptera: Noctuidae) e foram observados mortalidades na faixa de 55,0 a 70,0\%. O modo de ação dos ácidos $\omega$-fenil alcanoicos e alquenoicos em lepdópteras ainda é desconhecido.

Através do biomonitoramento realizado dos extratos e frações dos frutos de T. claussenii, pôde-se constatar que as substâncias ativas sobre $S$. frugiperda eram o conjunto dos ácidos $\omega$-fenil alcanoicos e alquenoicos. Esse conjunto de ácidos apresenta-se como uma nova classe química de potenciais agentes inseticidas, assim como os limonoides encontrados em meliáceas. ${ }^{16}$

\section{AGRADECIMENTOS}

Ao CNPq, à CAPES e FAPESP pelas bolsas e apoios financeiros concedidos.

\section{REFERÊNCIAS}

1. Cruz, I.; A lagarta-do-cartucho na cultura do milho, Embrapa/CNPMS: Sete Lagoas, 1995 (EMBRAPA-CNPMS, Circular Técnica, n. 21).

2. Carvalho, A. O. R.; Pragas do milho e seu controle, IAPAR: Londrina, 1982 (Circular Técnica, 29). 
3. Waquil, J. E.; Viana, P. A.; Lordello, A. I.; Pesq. Agropec. Bras. 1982, 17, 163; Bianco, R.; A cultura do milho no Paraná, IAPAR: Londrina, 1991 (Circular Técnica, 68).

4. Mikolajczak, K. L.; Reed, D. K.; J. Chem. Ecol. 1987, 13, 99.

5. Rodríguez, H. C.; Vendramim, J. D.; Man. Integ. Pragas 1996, 42, 14; Rodríguez, H. C.; Vendramim, J. D.; Rev. Agric. 1997, 72, 305.

6. Matos, A. P.; Nebo, L.; Batista-Pereira, L. G.; Vieira, P. C.; Fernandes, J. B.; Da Silva, M. F. G. F.; Rodrigues, R. R.; Bioassay 2006, 1/X, 1.

7. Matos, A. P.; Nebo L.; Vieira, P. C.; Fernandes, J. B.; da Silva, M. F. G. F.; Rodrigues, R. R.; Quim. Nova 2009, 32, 1553.

8. Pupo, M. T.; Vieira, P. C.; Fernandes, J. B.; Da Silva, M. F. G. F.; Phytochemistry 1996, 42, 795.

9. Pupo, M. T.; Vieira, P. C.; Fernandes, J. B.; Da Silva, M. F. G. F.; Rodrigues Fo., E.; Phytochemistry 1997, 45, 1495; Pupo, M. T.; Adorno, M. A. T.; Vieira, P. C.; Fernandes, J. B.; da Silva, M. F. G. F.; Pirani, J. R.; J. Braz. Chem. Soc. 2002, 13, 382.
10. Pupo, M. T.; Vieira, P. C.; Fernandes, J. B.; Da Silva, M. F. G. F.; Phytochemistry 1998, 48, 307.

11. Kasten, P. Jr.; Precetti, A. A. C. M.; Parra, J. R. P.; Rev. Agric. 1978, 53, 68; Parra, J. R. P. Em Controle microbiano de insetos; Alves, S. B., ed.; Editora Manole: São Paulo, 1986.

12. Paula, V. F.; Barbosa, L. C. A.; Demuner, A. J.; Piló-Veloso, D.; Picanço, M. C.; Pest Manag. Sci. 2000, 56, 168

13. Leonard, J.; Lygo, B.; Procter G.; Advanced Practical Organic Chemistry, $2^{\text {nd }}$ ed., Chapman \& Hall: Oxford, 1995.

14. Pizzolatti, M. G.; Verdi, L. G.; Brighente, I. M. C.; Nat. Prod. Res. 2004, $18,433$.

15. Vanderherchen, M. B.; Isherwood, M.; Thompson, D. M.; Linderman, R. J.; Roe, R. M.; Pest. Biochem. Physiol. 2005, 81, 71.

16. Champagne, D. E.; Koul, O.; Isman, M. B.; Scuder, G. G. E.; Towers, G. H. N.; Phytochemistry 1992, 31, 377; Rodriguez, B.; Caballero, C.; Ortego, F.; Castañera, P.; J. Nat. Prod. 2003, 66, 452. 